\title{
DO LUMBOSACRAL TRANSITIONAL ANOMALIES HAVE ANY RELATION WITH SAGITTAL SPINAL ALIGNMENT AND CORONAL SPINAL ASYMMETRY: A RETROSPECTIVE INVESTIGATION IN ARMY OFFICER CANDIDATES
}

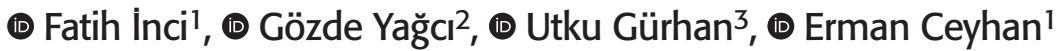 \\ ${ }^{1}$ University of Health Sciences Turkey, Ankara Bilkent City Hospital, Clinic of Orthopaedics and Traumatology, Ankara, Turkey \\ ${ }^{2}$ Hacettepe University Faculty of Health Sciences, Department of Physical Therapy and Rehabilitation, Ankara, Turkey \\ 3 Silopi State Hospital, Clinic of Orthopaedics and Traumatology, Şırnak, Turkey
}

Objective: Lumbosacral transitional vertebrae (LSTV) are commonly seen congenital anomalies of the lumbosacral spine, and named according to the transition type as sacralization or lumbarization. The aim of this study was to determine whether LSTV have influence on sagittal spinal alignment and coronal spinal asymmetry.

Materials and Methods: Anteroposterior and lateral full-length standing X-rays of young adult army officer candidates, who were admitted for health screening between January 2018 and January 2019, were extracted from the medical electronic database. Among these X-rays, those belonging to participants with sacralization and lumbarization were identified. The cervical lordosis, thoracic kyphosis and lumbar lordosis were measured on lateral X-rays and coronal spinal angle, if exist, were measured on anteroposterior X-ray with Cobb angle. Sagittal and coronal parameters were compared among participants with lumbarization, participants with sacralization and that of age-and sex-matched controls without LSTV.

Results: Of the 179 X-rays extracted from the database, 30 (16.8\%) were participants with sacralization, 69 (38.5\%) were participants with lumbarization and 90 (50.3\%) were controls. Participants with lumbarization had significantly greater cervical and lumbar lordosis angles than those without LSTV (controls). However, thoracic kyphosis angle did not differ among three groups. Based on coronal spinal measure, controls had higher spinal asymmetry values than participants with sacralization and participants with lumbarization. Curve patterns found in this study were single thoracic and single lumbar patterns.

Conclusion: The finding of this study demonstrated that individuals without LSTV were more likely to develop coronal spinal asymmetry. This study also suggested considering lumbarization for increase in physiological cervical and lumbar sagittal angles.

Keywords: Lumbarization, sacralization, sagittal spinal alignment, coronal spinal asymmetry, lumbosacral transitional vertebrae

\section{INTRODUCTION}

Lumbosacral transitional vertebrae (LSTV) are commonly seen congenital anomalies of the lumbosacral spine. The lumbosacral junction may be renamed according to the transition type: sacralization defines the assimilation of the fifth lumbar vertebra to the sacrum (fusion between the L4 and S1 segment), and lumbarization shows the transition of a sacral vertebra to a lumbar configuration (L6-S1). Men (4.7\% prevalence) are more likely to have transitional vertebrae compared to women $(2.7 \%$ prevalence) $)^{(1)}$. LSTV has been reported to be associated with low back-related symptoms. The presence of LSTV has been reported to be one of the factors for patients who apply with low back pain to orthopedics and traumatology departments in some clinical studies ${ }^{(2,3)}$. Some other studies have claimed that the LSTV anomaly may predispose patients to certain clinical disorders, such as smaller disk height, increased risk of lumbar disc herniation, early disc degeneration above the transitional segment, and spinal stenosis ${ }^{(4,5)}$. Sagittal and coronal spinal disorders are recognized as important in selecting army officer candidates in Turkey. Individuals with LSTV, or with sagittal spinal curves outside of the normal limits or coronal spinal asymmetry above $10^{\circ}$, will not be accepted for army officer positions as the rule.

Previously, abnormal torque moments at the vertebral segment above the transitional segment were reported as being responsible for disc degeneration ${ }^{(4)}$. Relative hypermobility 
above LSTV and the inability to distribute loads equally and, thereby, an increase in local stress in a vertebral segment is suggested to occur in the presence of LSTV(6). Price et al. ${ }^{(7)}$ showed alterations in spinopelvic parameters in subjects with LSTV. They reported increased pelvic tilt, pelvic incidence, sacral slope, and lumbar lordosis in subjects with lumbarization compared to healthy controls. Yokoyama et al. ${ }^{\left({ }^{8}\right)}$ showed increased spinopelvic parameters in asymptomatic individuals with lumbarization, like Price et al.(7), and they also found positive sagittal balance in that group of patients. These biomechanical changes with LSTV reported in the literature made us think that physiological sagittal curves might be affected by the LSTV and coronal spinal asymmetries may occur.

Lee et al. ${ }^{(5)}$ reported LSTV prevalence as $12.2 \%$ in adolescents with idiopathic scoliosis. The presence of LSTV has been considered important in determining surgical levels for the curve correction, because it affects spinal stability and compensation in idiopathic scoliosis ${ }^{(5)}$. Furthermore, Zhou et al.(1) found that LSTV significantly affected spinal alignment parameters, except for thoracic kyphosis and truncal tilt. They found an increase in the magnitude of sagittal pelvic (pelvic incidence and pelvic tilt), spinopelvic (sacral slope), and global spinal alignment (sagittal vertical axis, T1-pelvic angle, and lumbar lordosis) parameters.

We noticed the lack of retrospective studies that might help to define the relationship between LSTV and physiological sagittal spinal curves and the possible risk of coronal spinal asymmetry. The aim of this study was first to investigate whether sagittal spinal curves change in participants with LSTV, and, secondly, to analyze the relationship between LSTV and coronal spinal asymmetry. The third aim was to compare these spinal parameters between two different LSTV groups, namely participants with sacralization and participants with lumbarization.

\section{MATERIALS AND METHODS}

This study is retrospective radiographics analysis of hospital records, approval was obtained from the local ethics committee of University of Health Turkey, Ankara Bilkent City Hospital Ethics Board (date: 25.03.2020/16, decision no: 72300690790). The data of young adult army officer candidates in Turkey, who applied to the our hospital Department of Orthopedics and Traumatology for the purpose of health screening between January 2018 and January 2019, were extracted from the electronic database of the hospital. All anteroposterior and lateral radiographs were evaluated regarding the presence of LSTV.Among participants who had LSTV, those with sacralization or lumbarization were included in the study. Assimilation of the fifth lumbar vertebra to the sacrum was called sacralization, while transition of the first sacral vertebra to a lumbar configuration was called lumbarization ${ }^{(9)}$. In addition, an agematched control group with no signs of lumbosacral transitional anomalies was constituted. Participants were excluded if they had a history of spinal trauma/surgery, spondylolisthesis, spondylodiscitis, scoliosis, chronic inflammatory arthritis predominantly affecting the axial skeleton (e.g., ankylosing spondylitis, psoriatic arthritis), vertebral fracture, aseptic necrosis of the vertebra, and/or radiographs of inappropriate image quality.

On the standing full-length [36-in $(91-\mathrm{cm})]$ lateral radiograph of the spine, sagittal spinal curves including cervical lordosis, thoracic kyphosis, and lumbar lordosis and on the standing full-length anteroposterior radiograph, lateral curvature of the spine, if present, were measured with imaging software (RadiAnt DICOM Viewer version 5.5) using the Cobb method by a single examiner. Cervical lordosis was measured from vertebrae C2 through $C 7^{(10)}$. Thoracic kyphosis was measured in a similar manner using a line drawn along the superior endplate of $\mathrm{T} 1 / \mathrm{T} 2$ and the inferior endplate of $\mathrm{T} 11 / 12^{(10)}$. Lumbar lordosis was measured from the top of $L 1$ to the top of the sacrum ${ }^{(10)}$. For cervical and lumbar sagittal spinal measures, extension (lordosis) angles were considered as positive numbers, whereas flexion (kyphosis) angles were considered as negative numbers. For thoracic sagittal spinal measures, flexion (kyphosis) angles were considered as positive numbers.

Straight or symmetrical spines in the coronal plane were called normal spines in this study. Curves of $\geqslant 10^{\circ}$ in the coronal plane were accepted as scoliosis, whereas curves of less than $10^{\circ}$ were accepted as spinal asymmetry ${ }^{(11)}$. Curve patterns were classified according to the Scoliosis Research Society's classification ${ }^{(12)}$ They divided curves into single (thoracic or lumbar), double (both thoracic and lumbar), and triple patterns (upper thoracic, middle thoracic, and lumbar).

\section{Statistical Analysis}

Statistical analysis was performed with SPSS 15.0 for Windows (SPSS Inc., Chicago, IL, USA). The normality of the variables was checked by Kolmogorov-Smirnov test. Because the variables were normally distributed $(p>0.05)$, parametric tests were performed. One-Way ANOVA was used for betweengroup comparisons of continuous variables. For significant interactions, post-hoc pairwise comparisons were made using Tukey's method. The relationship between variables was assessed using Pearson's correlation analysis. Following Cohen's classification, the magnitude of the Pearson correlation coefficient was categorized as follows: 0.10 to 0.29 , low; 0.3 to 0.49 , moderate; and 0.5 or above, large ${ }^{(13)}$. The alpha level was 0.05 for all tests of statistical significance.

\section{RESULTS}

A total of 277 participants with both anteroposterior and lateral spine radiographs were identified from the electronic database. Of these participants, 254 who had proper radiographs were included in the study. After the assessment of the radiographs and medical records of these participants, 75 of them were excluded from the study for several different reasons as illustrated in the flowchart diagram (Figure 1). Analyses 
turkishspine

were undertaken of the remaining participants, including [1] participants with sacralization $(n=30),[2]$ participants with lumbarization ( $\mathrm{n}=59)$, and [3] age-matched control participants with no signs of lumbosacral transitional anomalies $(n=90)$.

The mean ages of participants with sacralization, with lumbarization, and with no signs of lumbosacral transitional anomalies were $18.8 \pm 1.2,18.8 \pm 1.2$, and $18.8 \pm 0.8$ years, respectively. There was no difference between the three groups regarding age $(p=0.988)$. Body mass index differed among the groups. Both participants with sacralization [mean difference, -0.86 (0.36); $95 \%$ confidence interval $(\mathrm{Cl}),-1.7$ to $-0.02, \mathrm{p}=0.043$ ] and lumbarization (mean difference, $-1.4(0.28) ; 95 \% \mathrm{Cl},-2.09$ to $-0.75, p<0.001$ ) had higher body mass indexes than the controls. Demographic characteristics are shown in Table 1.

For sagittal spinal alignment parameters, there was a difference between the three groups in cervical lordosis $(p=0.034)$ and

Subjects assessed for eligibility $(\mathrm{n}=277)$

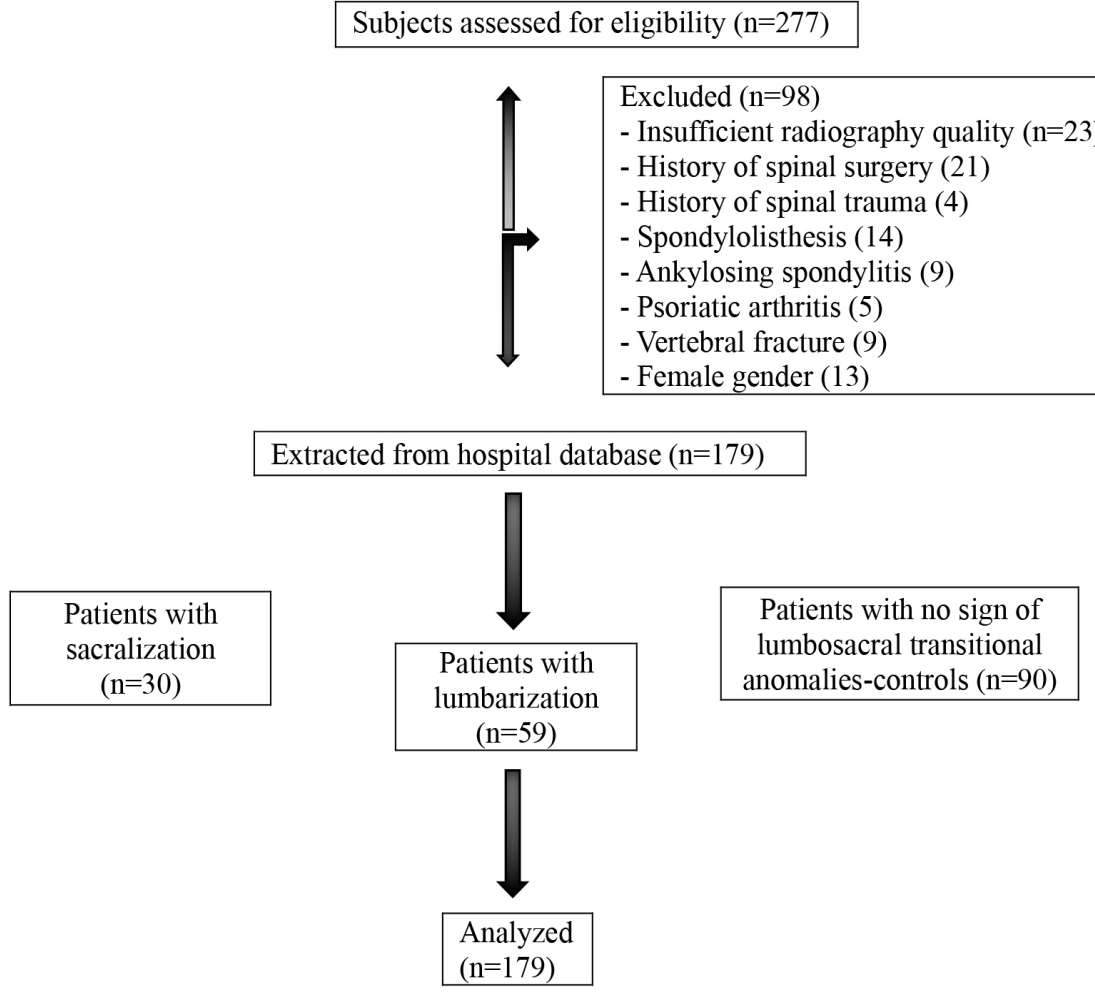

Figure 1. Flow diagram for participants of the study

Table 1. Comparison of demographic and clinical characteristics of the participants among groups

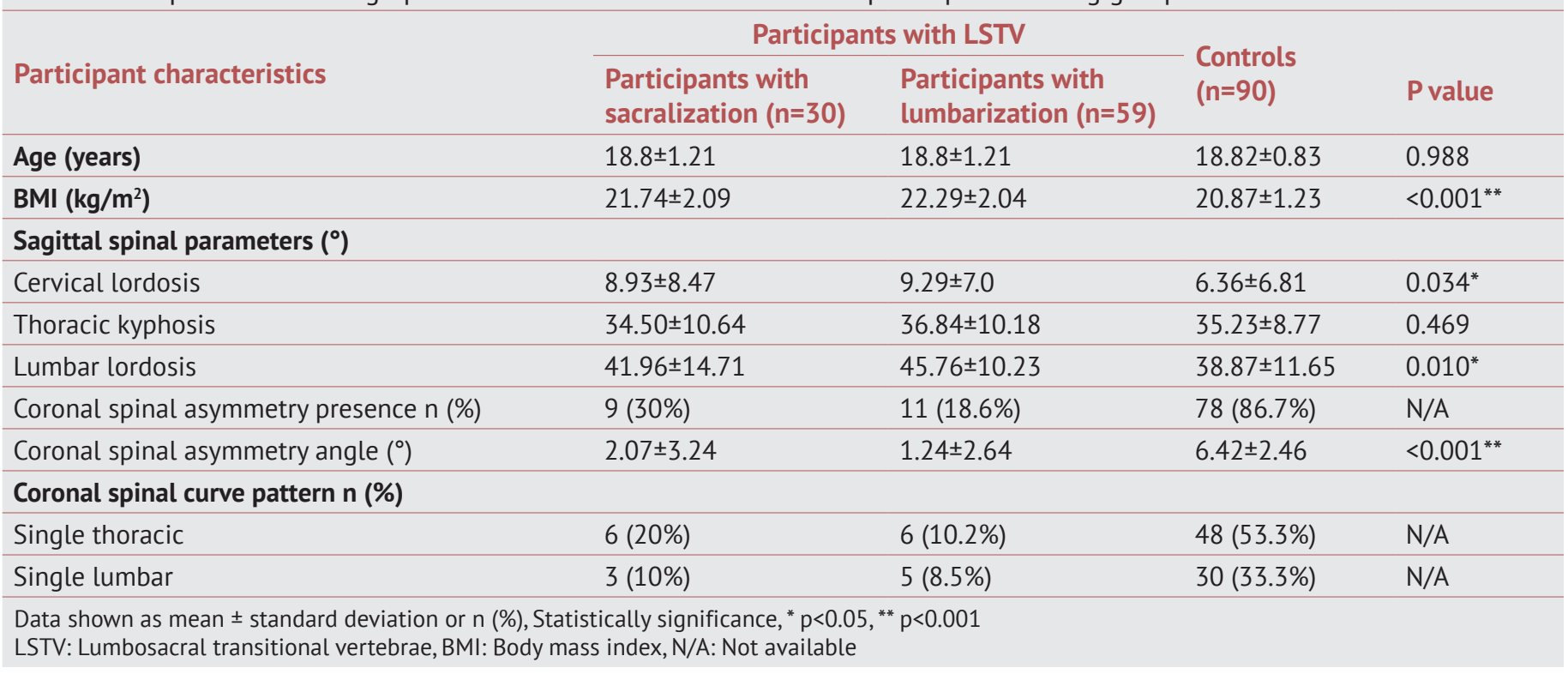


lumbar lordosis $(p=0.010)$ angles, whereas thoracic kyphosis did not differ among the groups $(\mathrm{p}=0.469)$ (Table 1$)$. Both cervical (mean difference, -2.93 (1.20); $95 \% \mathrm{Cl},-5.78$ to $-0.09, \mathrm{p}=0.041$ ) and lumbar lordosis (mean difference, -6.89 (2.25); $95 \% \mathrm{Cl}$, -12.2 to $-1.57, p=0.007$ ) angles were greater in participants with lumbarization than in controls.

Based on coronal spinal measures, there were participants with spinal asymmetry in all groups, whereas there was no participant with scoliosis. However, spinal asymmetry intensity differed among groups $(p<0.001$ ) (Table 1 ). Pairwise comparisons revealed that controls had higher spinal asymmetry values than participants with sacralization (mean difference, 4.35 (0.56); $95 \% \mathrm{Cl}, 3.02$ to $5.67, \mathrm{p}<0.001)$ and participants with lumbarization (mean difference, $5.18(0.44) ; 95 \% \mathrm{Cl}, 4.13$ to $6.24, p<0.001$ ). Curve patterns found in this study were single thoracic and single lumbar

Correlation analysis established that coronal spinal asymmetry negatively correlated with cervical lordosis and lumbar lordosis and positively correlated with thoracic kyphosis. Both cervical lordosis and thoracic kyphosis were positively correlated with lumbar lordosis. Correlations were found to be low or moderate. Correlations between sagittal spinal parameters and coronal spinal asymmetry are summarized in Table 2 .

\section{DISCUSSION}

This paper explores whether sagittal spinal curves change in young male participants with LSTV, analyzes the relationship between LSTV and coronal spinal asymmetry, and compares sagittal and coronal spinal parameters between two different LSTV groups, namely participants with sacralization and participants with lumbarization. The study showed that participants with lumbarization had greater cervical and lumbar lordosis angles than controls without LSTV. Presence of LSTV was not seen to affect the thoracic kyphosis angle. Interestingly, controls without LSTV were found to have higher spinal asymmetry prevalence and mean values than participants with LSTV.

According to Panjabi(13) ${ }^{(13)}$ the mechanical stability of the spine is necessary to maintain spinal functions such as load carrying and core stabilizing for movements of the extremities. The spinal stabilizing system consists of active (muscles and tendons surrounding the spinal column), passive (vertebrae, facet articulations, intervertebral discs, spinal ligaments, joint capsules, passive mechanical properties of the muscles), and neural (various force and motion transducers, located in ligaments, tendons, and muscles, and the neural control centers) subsystems. Both hypomobility and hypermobility of the spine as measured by the range of motion have been reported as predisposing factors of spinal instability. When spinal stability is deteriorated, the capacity in resisting torsional loads is reported to weaken and compensatory changes occur ${ }^{(13)}$. We determined in the present study that, compared to individuals without LSTV, those with lumbarization presented with different sagittal alignment. As expected, with an extra lumbar vertebra, lumbar lordosis was on average $7^{\circ}$ greater in subjects with lumbarization than controls. In the presence of lumbarization, separation of the first sacral segment from the sacral corpus results in the number of lumbar vertebrae increasing to six. Lumbarization could probably result in a more mobile lumbar segment, thereby increasing the physiological lumbar curve. Similarly, Price et al. ${ }^{(7)}$ found an average $8^{\circ}$ increase in patients with lumbarization when compared with an asymptomatic population: Yokoyama et al..$^{(8)}$ also showed that lumbar lordosis tended to be greater by an average of $3^{\circ}$ in individuals with lumbarization. They noted positive sagittal balance when lumbarization occurred. In the present study, there was an increase in cervical lordosis of about $3^{\circ}$ in the lumbarization group, whereas thoracic kyphosis did not differ among groups. Similarly, Zhou et al. ${ }^{(1)}$ found that LSTV significantly affected lumbosacral sagittal spinal alignment parameters, but no difference in the physiological curvature of the spine above the lumbar spine existed between patients with LSTV and healthy controls. Alterations in structures resulting in biomechanical adjustments of these segments may change the muscular efforts around the spine in order to achieve optimal movement without compromising stability.

Previously, possible variations in the number of thoracic and lumbar vertebrae were investigated in individuals with adolescent idiopathic scoliosis because of their importance in surgical correction ${ }^{(14)}$. Atypical vertebral anatomy has been proposed as a risk factor for wrong-site spine surgery in coronal spinal deformities and it was suggested that vertebrae variation occurs with considerable frequency ( $10 \%$ of prevalence) in this population. Lee et al.(5) suggested considering LSTV with $12.2 \%$ prevalence in patients with adolescent idiopathic scoliosis. On the other hand, Seçer et al. ${ }^{(15)}$ transitional vertebra detected in $18(4.5 \%)$ of a total of 401 young male patients with low back

Table 2. Correlation between sagittal spinal parameters, coronal spinal asymmetry and body mass index

\begin{tabular}{lcccc}
\hline \multirow{2}{*}{ N=179 } & \multicolumn{3}{c}{ All participants } \\
\cline { 2 - 5 } & Cervical lordosis & Thoracic kyphosis & Lumbar lordosis & Coronal spinal asymmetry \\
\hline Cervical lordosis & 1 & $r=-0.031, p=0.676$ & $r=0.229, p=0.002^{*}$ & $r=-0.260, p<0.001^{* *}$ \\
\hline Thoracic kyphosis & 1 & $r=0.201, p=0.997^{*}$ & $r=0.219, p=0.003^{*}$ \\
\hline Lumbar lordosis & & 1 & $r=0.186, p=0.013^{*}$ \\
\hline Significance is indicated by $p$-values; ${ }^{*} p<0.05,{ }^{* *} p<0.001$ & & &
\end{tabular}


pain without scoliosis. In contrast, higher spinal asymmetry values were seen in controls without LSTV than participants with LSTV (both in sacralization and lumbarization groups) in the present study. Comparative studies investigating the relation between coronal spinal asymmetry and LSTV are limited. Given the methods used in the current study, it is not possible to determine which mechanisms were responsible for the high prevalence of coronal spinal asymmetry in controls without transitional vertebrae.

Kiel et al.(9) defined the alteration of sagittal curves in patients with low magnitude idiopathic scoliosis as coronal spinal asymmetry (average Cobb angle of $5^{\circ}$ ). They reported backward vertebral tilt between $\mathrm{T} 7$ and $\mathrm{L} 3$ and forward tilt at T5 and L5. Segmental sagittal angulation was found to be more common in patients with greater Cobb angles ${ }^{(9)}$. Clement et al. found that the low kyphosis in idiopathic scoliosis has a trend of accompanying low lordosis(16). Yu et al. ${ }^{(17)}$ highlighted that the sagittal cervical angle is correlated with global sagittal and coronal alignment in young patients with idiopathic scoliosis. They emphasized that cervical kyphosis was related to coronal spinal deviation. On the contrary, Hu et al.(18) found that coronal and sagittal parameters were not significantly correlated, and the coronal deformity types did not change the global sagittal postural patterns. Concerning coronal and sagittal spinal parameters, our study identified an association between coronal spinal asymmetry (Cobb angle below $10^{\circ}$ ) and sagittal spinal parameters. Coronal spinal asymmetry was negatively correlated with cervical lordosis and lumbar lordosis and positively correlated with thoracic kyphosis. In addition, both cervical lordosis and thoracic kyphosis were positively correlated with lumbar lordosis. These relations may be explained by the flexible spine characteristics of young people and the spontaneous adaptation mechanism of the spinalpelvic system.

\section{Study Limitations}

The present study has some limitations. First, this study had a retrospective design. Second, the subject population consisted of young male participants. Therefore, results cannot be generalized to other age groups or to females. Body mass index distribution among the groups was different. Clinical measurements such as pain, function, and health-related quality of life of the participants might have been beneficial to explain the relations between the findings of this study.

\section{CONCLUSION}

The results of this study showed that young male participants with lumbarization had increased cervical and lumbar lordosis curves in the sagittal plane compared to controls without LSTV, whereas thoracic kyphosis was not affected by the presence of transitional vertebrae. LSTV was not found to have any effect on the risk of coronal spinal asymmetry development in this study. Therefore, the detailed investigation of the relation between LSTV and sagittal spinal alterations and factors that might affect this relationship is suggested for future studies, because these could cause symptoms in the long term.

\section{Ethics}

Ethics Committee Approval: Universtiy of Health Sciences Turkey, This study was approved by the local ethics committee of Ankara Bilkent City Hospital Ethics Board (date: 25.03.2020/16, decision no: 72300690-790).

Informed Consent: The patients were informed regarding the treatment and its potential benefits as well as evaluation methods; and thereby, signed informed consent forms were obtained.

Peer-review: Internally peer-reviewed.

\section{Authorship Contributions}

Surgical and Medical Practices: F.I., U.G., E.C., Concept: F.I., G.Y., U.G., Design: G.Y., U.G., E.C., Data Collection or Processing: F.I., U.G., Analysis or Interpretation: G.Y., E.C., Literature Search: F.I., G.Y., U.G., E.C., Writing: F.I., G.Y., U.G., E.C.

Conflict of Interest: No conflict of interest was declared by the authors.

Financial Disclosure: The authors declared that this study received no financial support.

\section{REFERENCES}

1. Zhou PL, Moon JY, Tishelman JC, Errico TJ, Protopsaltis TS, et al. Interprtation of spinal radiographic parameters in patients with transitional lumbosacral vertebrae. Spine Deform. 2018;6:587-92.

2. Kara GK, Kavak H. Does lumbosacral transitional vertebrae cause low back pain. J Turk Spinal Surg. 2020;31:234-8.

3. Quinlan JF, Duke D, Eustace S. Bertolotti's syndrome: a cause of back pain in young people. J Bone Joint Surg Br. 2006;88:1183-6.

4. Castellvi AE, Goldstein LA, Chan DP. Lumbosacral transitional vertebrae and their relationship with lumbar extradural defects. Spine (Phila Pa 1976). 1984;9:493-5.

5. Lee CS, Ha JK, Kim DG, Hwang CJ, Lee DH, Cho JH. The clinical importance of lumbosacral transitional vertebra in patients with adolescent idiopathic scoliosis. Spine (Phila Pa 1976). 2015;40:964-70.

6. Bron JL, van Royen BJ, Wuisman PI. The clinical significance of lumbosacral transitional anomalies. Acta Orthop Belg. 2007;73:687-95

7. Price R, Okamoto $M$, Le Huec JC, Hasegawa K. Normative spinopelvic parameters in patients with the lumbarization of $\mathrm{S} 1$ compared to a normal asymptomatic population. Eur Spine J. 2016;25:3694-8.

8. Yokoyama K, Kawanishi M, Yamada M, Tanaka H, Ito Y, Kawabata S, et al. Spinopelvic alignment and sagittal balance of asymptomatic adults with 6 lumbar vertebrae. Eur Spine J. 2016;25:3583-8.

9. Kiel A, Burwell RG, Moulton A, Purdue M, Webb JK, Wojcik AS Segmental patterns of sagittal spinal curvatures in children screened for scoliosis: kyphotic angulation at the thoracolumbar region and the mortice joint. Clinical Anatomy. 1992;5:353-71.

10. Harrison DE, Cailliet R, Harrison DD, Janik TJ, Holland B. Reliability of centroid, Cobb, and Harrison posterior tangent methods: which to choose for analysis of thoracic kyphosis. Spine (Phila Pa 1976). $2011 ; 26: 227-34$

11. Van Goethem J, Van Campenhout A, van den Hauwe L, Parizel PM Scoliosis. Neuroimaging Clin N Am. 2007;17:105-15.

12. Donzelli S, Poma S, Balzarini L, Borboni A, Respizzi S, Villafane $\mathrm{H}$, et al. State of the art of current 3-D scoliosis classifications: a 
systematic review from a clinical perspective. J Neuroeng Rehabil. 2015; 16;12:91.

13. Panjabi MM. The stabilizing system of the spine. Part I. Function, dysfunction, adaptation, and enhancement. J Spinal Disord. 1992;5:383-9.

14. Ibrahim DA, Myung KS, Skaggs DL. Ten percent of patients with adolescent idiopathic scoliosis have variations in the number of thoracic or lumbar vertebrae. J Bone Joint Surg Am. 2013;1;95:828-33

15. Seçer M, Muradov JM, Dalgiç A. Evaluation of congenital lumbosacral malformations and neurological findings in patients with low back pain. Turk Neurosurg. 2009;19:145-8.
16. Clément JL, Geoffray A, Yagoubi F, Chau E, Solla F, Oborocianu I, et al. Relationship between thoracic hypokyphosis, lumbar lordosis and sagittal pelvic parameters in adolescent idiopathic scoliosis. Eur Spine J. 2013;22:2414-20.

17. Yu M, Silvestre C, Mouton T, Rachkidi R, Zeng L, Roussouly P. Analysis of the cervical spine sagittal alignment in young idiopathic scoliosis: a morphological classification of 120 cases. Eur Spine J. 2013;22:2372-81.

18. Hu P, Yu M, Liu X, Zhu B, Liu X, Liu Z. Analysis of the relationship between coronal and sagittal deformities in adolescent idiopathic scoliosis. Eur Spine J 2016;25:409-16. 